\title{
The influence of the aspheric profiles for transition zone on optical performance of human eye after conventional ablation
}

L. Fang

fanglh71@126.com

\section{Key Laboratory of Nondestructive Test (Ministry of Education), Nanchang Hangkong University,} Nanchang, 330063, China

The analysis in the impact of transition zone on the optical performance of human eye after laser refractive surgery is important for improving visual correction technology. By designing the ablation profiles of aspheric transition zone and creating the ablation profile for conventional refractive surgery in optical zone, the influence of aspheric transition zone on residual aberrations was studied. The results indicated that the ablation profiles of transition zone had a significant influence on the residual wavefront aberrations. For a hyperopia correction, the profile \#9 shows a larger induced coma and spherical aberration when the translation of the centre of pupil remains constant. However, for a myopia astigmatism correction, the induced coma and spherical aberration in profile \# 1 shows relatively larger RMS values than those in other profiles. Therefore, the residual higher order aberrations may be decreased by optimizing ablation profiles of transition zone, but they cannot be eliminated. In order to achieve the best visual performance, the design of ablation pattern of transition zone played a crucial role.

[DOI: http://dx.doi.org/10.2971/jeos.2014.14060]

Keywords: Aspheric transition zone, wavefront aberration, conventional ablation

\section{INTRODUCTION}

To deliver a conventional correction, it is important to understand the residual wavefront aberrations induced by ablation profiles. This paper concerns the effect of aspheric transition zone on residual optical aberrations after a conventional corneal ablation.

Transition zone, which connects the optical zone to the unaltered cornea, is included in modern laser systems for refractive surgery [1]. Transition zone can ensure that the curvature is continuous at the boundary between transition zone and untreated cornea and at the boundary between optical zone and transition zone. The use of transition zone during LASIK resulted in a low incidence of postoperative glare and halos [2]. In addition, a transition zone was used in photorefractive keratectomy for high myopia [3]. However, the exact size, shape and the profile of transition zone have profound impact on the residual aberrations. Some researches proved that the aspheric transition zone was safe and predictable [4, 5]. Arbelaez et al. reported that a multidynamic aspheric transition zone was included in a laser system in order to minimize the amount of induced aberrations [6]. Our research revealed that the transition zone had a significant impact on residual wavefront aberration [7]. So the effect of the ablation profile of transition zone on the residual aberrations deserves further study and research in this theoretical analysis.

The subclinical treatment decentration often occurs during corneal refractive surgery and the decentration has been observed in many researches. These results indicated that the centration errors exerted a significant influence on the residual wavefront aberrations [8]. Decentrations during refractive surgery may account for a portion of the postoperative increases in higherorder aberrations $[9,10]$. On the other hand, lateral decentration produced significantly greater residual higher-order aberrations RMS values than cyclotorsional misalignment during wavefront-guided excimer laser corneal ablation [11]. Therefore, the lateral decentration was only taken into account in this study. Theoretically, the translation of ablation profile could be simulated by coordinate transformation.

Until now the influence of aspheric transition zone on optical performance of human eye with consideration of treatment decentration for a conventional surgery has not been explicitly evaluated yet. In this paper, based on the corneal ablation profile, we evaluated the relationship between the predicted residual aberrations and the ablation profiles for transition zone. The impact of oblique incidence was also taken into account.

\section{MATERIALS AND METHODS}

\subsection{Ablation profile for conventional laser refractive surgery}

In general, myopia and astigmatism components are generally included in the refractive errors in human eyes. Based on the Munnerlyn equation, the ablation depth of cornea can be determined as shown in Figure 1 when the anterior corneal surface is a spherical surface after corneal refractive surgery. In the present research, the ablation profile for conventional 
myopia astigmatism correction can be given by [12]:

$$
\begin{aligned}
D_{s}(x, y)= & \sqrt{\left(\sqrt{R_{i x}^{2}-x^{2}}+R_{i y}-R_{i x}\right)^{2}-y^{2}} \\
& -\sqrt{R_{f}^{2}-x^{2}-y^{2}}+\sqrt{R_{f}^{2}-\left(O_{z} / 2\right)^{2}} \\
& +R_{i x}-R_{i y}-\sqrt{R_{i x}^{2}-\left(O_{z} / 2\right)^{2}}
\end{aligned}
$$

Here, $R_{i x}$ and $R_{i y}$ depict the radii of curvature of two principal meridians of the anterior corneal surface before refractive surgery. Also, $R_{f}$ represents the postoperative radius of curvature. $O_{z}$ is the diameter of optical zone in $x$-axis.

In practical application, the values of $R_{f}, R_{i x}$ and $R_{i y}$ for myopia astigmatism correction in individual human eyes can be calculated from the measured $\mathrm{k}$ value of cornea from corneal topographer and the subjective refraction in pupil plane as Ref [12].

\subsection{Ablation profile of aspheric transition zone}

In this study, the ablation profile for transition zone should be restructured, which was required to produce a smooth transition from the optical zone edge to the unaltered cornea. Aspheric ablation algorithm has been proved to be safe and predictable. An aspheric ablation profile is restructured as follows:

$$
d(x, y)=f_{b}(x, y) \cdot d_{e}(x, y)
$$

Here

$$
d_{e}(x, y)=f\left(\frac{O_{z} x}{2 \sqrt{x^{2}+y^{2}}}, \frac{O_{z} y}{2 \sqrt{x^{2}+y^{2}}}\right)
$$

It indicates the extended ablation depth in transition zone, which is extended from the boundary value of the optical zone. Here, $\mathrm{O}_{z}$ indicates the diameter of optical zone.

In addition, $f_{b}(x, y)$ represents as a blend function being aspheric ablation algorithm. It can give the normalized ablation depth in transition zone. The function value is one at the boundary between the optical zone and the transition zone, but the value changes to be zero at the boundary between the transition zone and the untreated periphery. Before the blend function is achieved, an aspheric ablation function must be created as follows:

$$
f_{b}^{\prime}(x, y)=h-\frac{\left(x^{2}+y^{2}\right)}{R+\left[R^{2}-\left(x^{2}+y^{2}\right)(Q+1)\right]^{1 / 2}}
$$

or

$$
f_{b}^{\prime}(x, y)=\frac{\left[x_{0}-\left(x^{2}+y^{2}\right)^{1 / 2}\right]^{2}}{R+\left\{R^{2}-\left[x_{0}-\left(x^{2}+y^{2}\right)^{1 / 2}\right]^{2}(Q+1)\right\}^{1 / 2}}
$$

Here, $h$ represents the nonzero value of ablation depth at the optical zone edge and $x_{0}$ conveys the width of transition zone. $Q$ depicts the asphericity. Also, $R$ conveys the radius of curvature of the ablation profile. The value can be computed as follows:

$$
R=\frac{\left(x_{0}\right)^{2}+h^{2}(1+Q)}{2 h}
$$

Then, the aspheric blend function can be obtained through normalization.

$$
f_{b}(x, y)=f_{b}^{\prime}(x, y) / h
$$

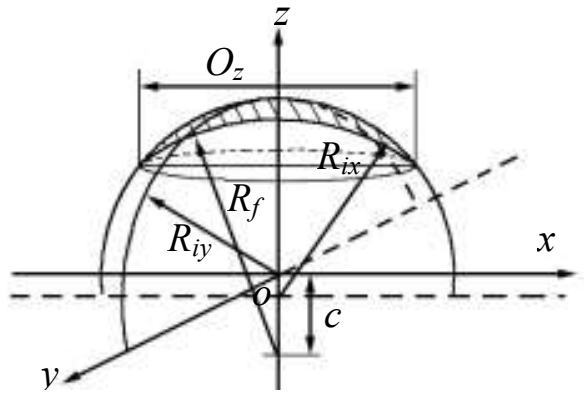

FIC. 1 Corneal shape and tissue ablation depth for conventional laser refractive surgery with the curvature radii of the corneal anterior surface after surgery $R_{f}$ and before surgery $R_{i x}$ and $R_{i y}$ and the diameter of the optical zone $\mathrm{O}_{z}$.

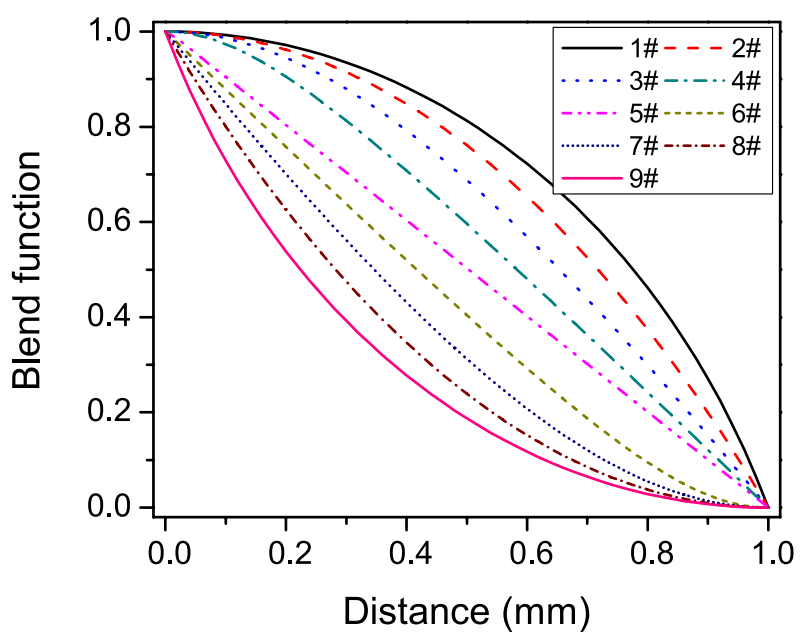

FIG. 2 Nine types of aspheric ablation profiles for transition zone. The corresponding $Q$ values for the profiles from profile \#1 to profile \#5 are $40,5,-30,-65$ and -100 respectively, which are calculated from Eq. (4). Similarly, the corresponding $Q$ values for the profiles from profile \#6 to profile \#9 are $-65,-30,5$ and 40 respectively, which are calculated from Eq. (5). Here, $h=0.1 \mathrm{~mm}$ and the width of transition zone is $1.05 \mathrm{~mm}$. Each curve shows the normalized ablation depth over transition zone from Eq. (7).

In this study, we have developed nine types of ablation profiles (from \#1 to \#9) by changing the asphericity ( $Q$ value). Then, the relationship of the normalized aspheric blend function versus transition zone width is shown in Figure 2. The vertical axis depicted the normalized ablation depth and the horizontal axis showed the normalized vertical distance from the ablating point in transition zone to the optical zone edge.

\subsection{Residual optical aberrations from ablation profile including transition zone}

According to the ablation profile including transition zone for pure myopic and hyperopic correction, the theoretical ablation depth of cornea in whole ablation zone can be calculated. When decentration occurs, the actual ablation depth can be computed by coordinate transformation. In addition, the refractive surgery lasers scan the ablation zone by angular projection. If the distance from the ablation plane to the scanner mirror is large, the incidence angle of laser beam onto a flat surface perpendicular to the axis of the laser is near zero. Therefore, the beam can be considered as moving vertically parallel to the center axis of human eye in refractive surgery. 
Consequently, the incidence angle $(\alpha)$ of laser beam on cornea is deduced from the mathematical model of anterior corneal surface. Then the adjustment factor of the ablation depth of cornea in ablation zone is determined by the reflection losses of laser energy and geometrical distortions. Finally, the actual ablation depth can be calculated by multiplying the above adjustment factor when laser oblique incidence is taken into account. Finally, the ablation depth can be converted into the actual corrected aberrations and the corresponding Zernike coefficients can be obtained by surface fitting.

$$
W_{e}=\sum_{n \text { and } m} C_{n}^{m} Z_{n}^{m}
$$

Then, the predicted residual aberrations are calculated as follows:

$$
\begin{aligned}
W_{r}(x, y) & =W_{p}(x, y)+W_{e}(x, y) \\
& =\sum_{n \text { and } m} a_{n}^{m} Z_{n}^{m}(x, y)+\sum_{n \text { and } m} C_{n}^{m} Z_{n}^{m}(x, y)
\end{aligned}
$$

Here, $C_{n}^{m}$ is the actual corrected Zernike coefficient with decentration, and $a_{n}^{m}$ is the coefficient of the original lower-order aberrations.

\section{RESULTS}

\subsection{Induced optical aberrations for pure hyperopic correction}

We calculated the theoretical ablation depth in the whole ablation zone including transition zone according to the ablation profile for conventional correction. The translation of the ablation centre was simulated by coordinate transformation. The impact of oblique incidence on ablation depth of cornea was evaluated by an adjustment factor [12]. Then the ablation depth was converted into the actual corrected aberrations. The induced aberrations were represented as the differences between the original lower-order aberrations and actual corrected aberrations. The relationship between the induced aberrations and the amount of decentration in $x$-axis and the types of ablation profile in transition zone was studied. Here, the original and induced aberrations are all up to sixth order and the diameter of the optical zone is $6 \mathrm{~mm}$. In addition, the width of transition zone $x_{0}$ is $1.05 \mathrm{~mm}$, and then the diameter of ablation zone is up to $8.1 \mathrm{~mm}$. Clinically, myopia and astigmatism components are generally included in the refractive errors of the human eyes. But when the root mean square (RMS) of astigmatism is lower, the ablation profile for pure myopic is usually applied in the conventional refractive surgery. Furthermore, because the ablation depth of every point at the edge of optical zone for pure myopic correction (rotational symmetry) is zero, the types of ablation profile in transition zone have no effect on the induced aberrations in this research. Therefore, only the relationships among the induced higher-order aberrations, the types of ablation profile and the amount of decentration for correcting pure hyperopia were discussed. The results are shown in Figure 3 when the refractive error is pure hyperopia being $+3 \mathrm{D}$.

In addition, considered the defocus with rotational symmetry, no optical aberrations were induced from eye cyclotorsion for pure myopia or hypermetropia correction. Therefore, the effect of eye cyclotorsion was not considered. The results show that the main induced aberrations are coma $\left(C_{31}\right)$ and spherical aberration $\left(C_{40}\right)$. Additionally, the induced trefoil $\left(C_{33}\right)$, secondary astigmatism $\left(C_{42}\right)$, secondary coma $\left(C_{51}\right)$ and secondary sphere $\left(C_{60}\right)$ also have relatively large RMS values. Other aberration terms have relatively small RMS values and ranged from 0 to $0.5 \mu \mathrm{m}$. In addition, the Figure 4 shows the relationship between the residual aberrations and the types of ablation profile of aspheric transition zone for correcting pure hyperopia being $+3 \mathrm{D}$ when the translation of the centre of pupil remains constant being $0.4 \mathrm{~mm}$.

In Figure 3(a), the results indicate that the profile \#1 shows the increasing induced trefoil as the increase of translation and the profile \#9 shows little impact of translation on the induced trefoil and its RMS value is smaller. From Figure 3(b), the induced coma is proportional to the translation of the centre of pupil and the profile \#9 shows a larger induced coma. The horizontal coma is induced from the translation in $x$-axis. Figure 3(c) indicates that the induced secondary astigmatism is closely correlated with the ablation profiles of transition zone. The induced secondary astigmatism for profile \#1 increases as the increase of the translation of the centre of pupil. But for profile \#9, it increases first, and then decrease with increasing translation. Figure 3(d) shows that the postoperative spherical aberration increases theoretically with the increase of translation and its sign for hyperopia correction is negative. The profile \#9 shows that the induced spherical aberration has a faster increase than in other profiles. From Figure 3(e) and 3(f), the induced secondary coma or secondary sphere for profile \#1 is nearly proportional to the translation of the centre of pupil, and yet it increases first, and then decrease as the increase of translation for profile \#9. In addition, other aberration terms are correlated slightly with the value of translation.

Additionally, Figure 4 reveals that the induced residual coma, spherical aberration and secondary coma increase quickly with the number of ablation profile for transition zone. However, some other aberration terms do not change with the increase of the number of ablation profile, such as trefoil, tetrafoil, pentafoil, secondary trefoil and so on.

\subsection{Induced optical aberrations for myopia astigmatism correction}

In clinical practice, most of the myopic eyes suffer from not only the myopic component but also the astigmatism component of refractive errors. The theoretical corneal ablation depth can be calculated from the ablation profile for myopia astigmatism correction. Similarly, the transverse translation is simulated by coordinate transformation. The induced aberrations are calculated as the difference between the original lower-order aberrations and actual corrected aberrations. The results have shown that the induced aberrations include almost all Zernike aberration terms. Similarly, the main terms are trefoil $\left(C_{33}\right)$, coma $\left(C_{31}\right)$, secondary astigmatism $\left(C_{42}\right)$, spherical aberration $\left(C_{40}\right)$ and secondary coma $\left(C_{51}\right)$. The Figure 5 shows the contour maps of the induced higher-order aberrations. In like manner, the diameter of the optical zone is $6 \mathrm{~mm}$ and the width of transition zone $x_{0}$ is $1.05 \mathrm{~mm}$. 

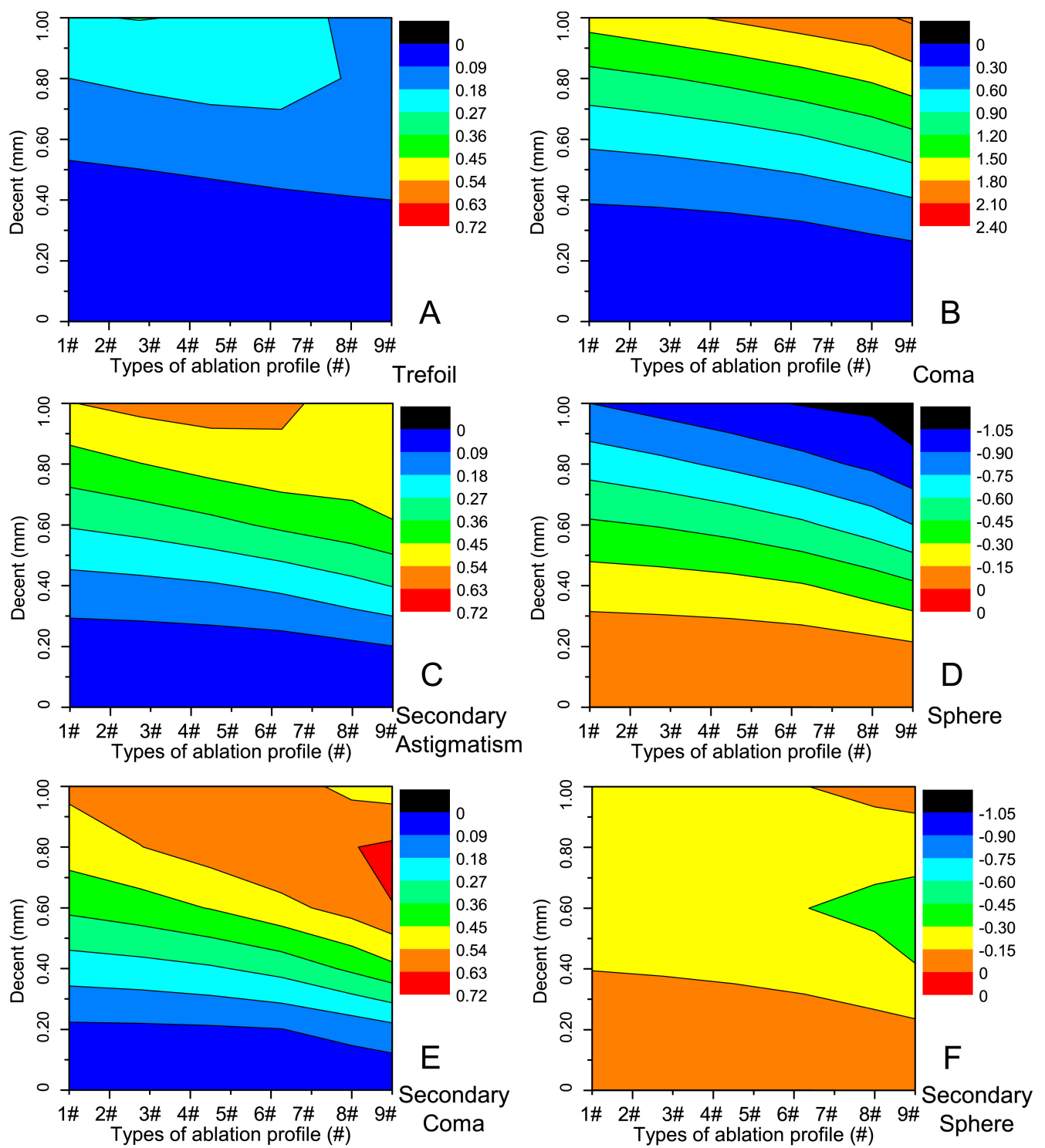

FIG. 3 The contour maps of the induced higher-order aberrations for correcting pure hyperopia being +3D. The diameter of the optical zone is 6 mm. The width of transition zone $x_{0}$ is $1.05 \mathrm{~mm}$ and the ablation zone diameter is up to $8.1 \mathrm{~mm}$. The results show that the main induced aberrations are coma $\left(C_{31}\right)$ and spherical aberration $\left(C_{40}\right)$.

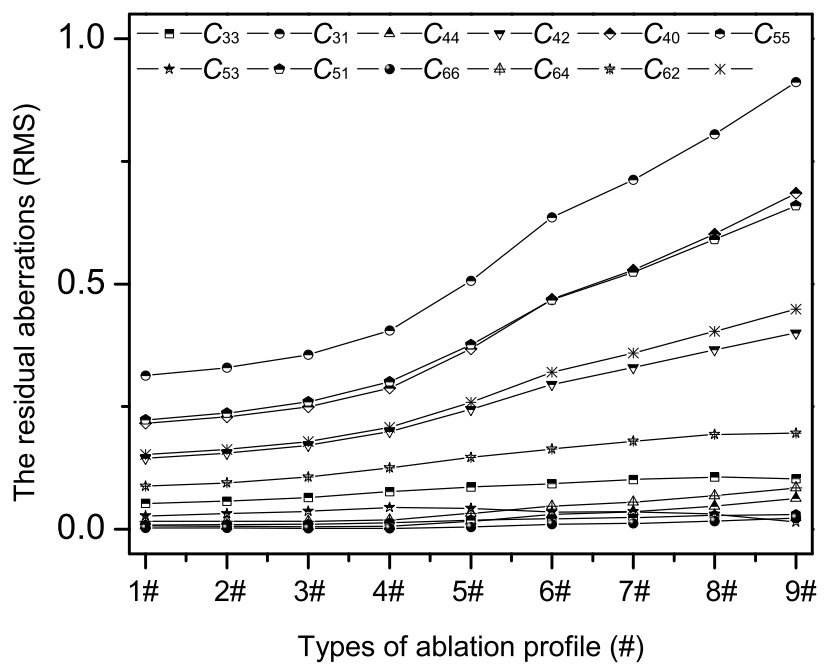

FIG. 4 The residual higher-order aberrations for correcting pure hyperopia being $+3 \mathrm{D}$ versus the types of ablation profile of transition zone. The diameter of the optical zone is $6 \mathrm{~mm}$ and the width of transition zone $x_{0}$ is $1.05 \mathrm{~mm}$. The results indicate the induced residual coma, spherical aberration and secondary coma are correlated closely with the ablation profile for transition zone. 

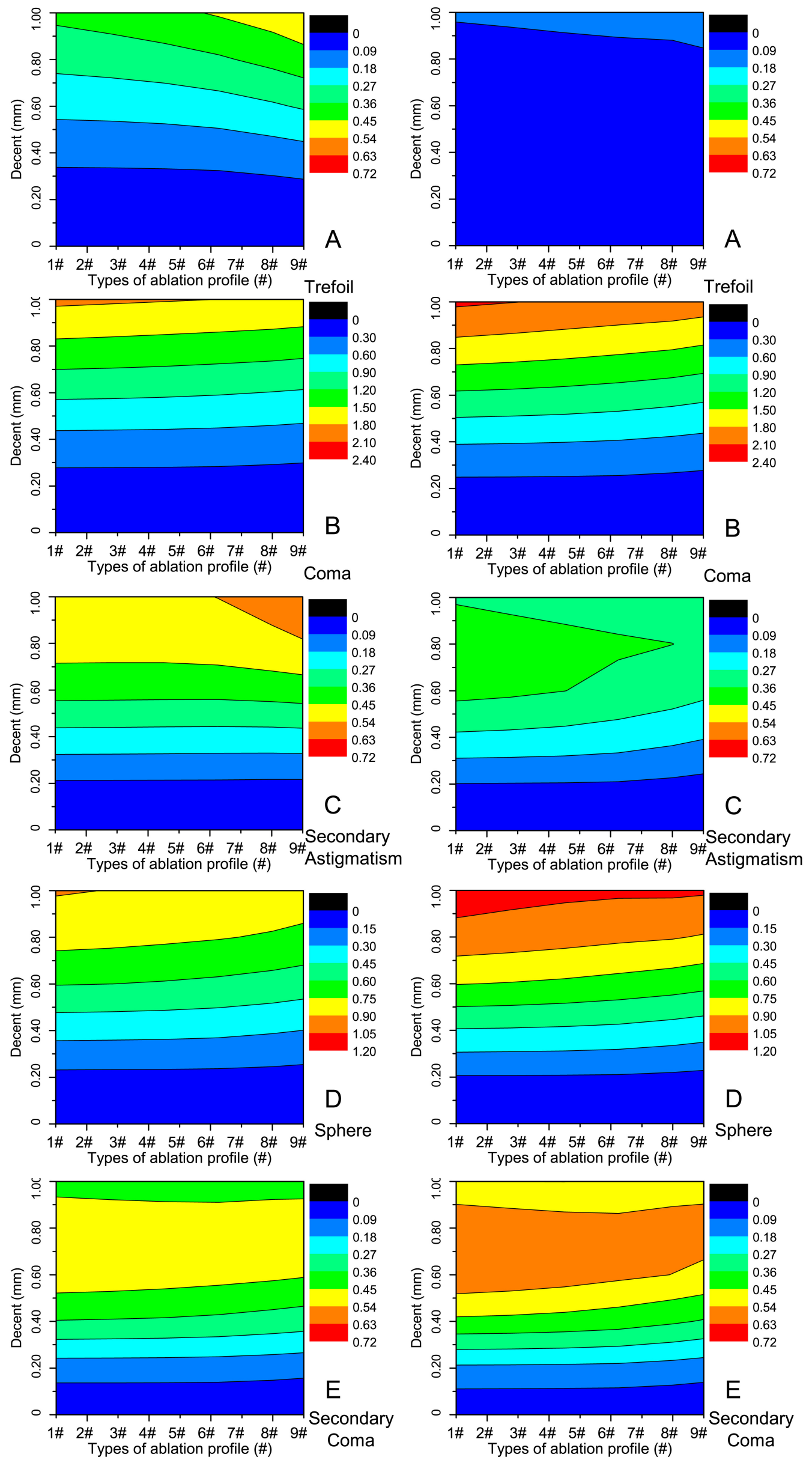

(a) For $-5.0 \mathrm{DS}-2.0 \mathrm{DC} \times 60^{\circ}$

(b) For $-7.5 \mathrm{DS}-1.5 \mathrm{DC} \times 180^{\circ}$

FIG. 5 The contour maps of the induced higher-order aberration for correcting myopia astigmatism. The panel (a) corresponds to the refractive errors $\left(-5.0 \mathrm{DS}-2.0 \mathrm{DC} \times 60^{\circ}\right)$ and the panel (b) corresponds to another refractive errors $\left(-7.5 \mathrm{DS}-1.5 \mathrm{DC} \times 180^{\circ}\right)$. The diameter of the optical zone is $6 \mathrm{~mm}$. The results show that the induced coma and sphere for profile \# 1 are relatively larger than those for profile \#9. 


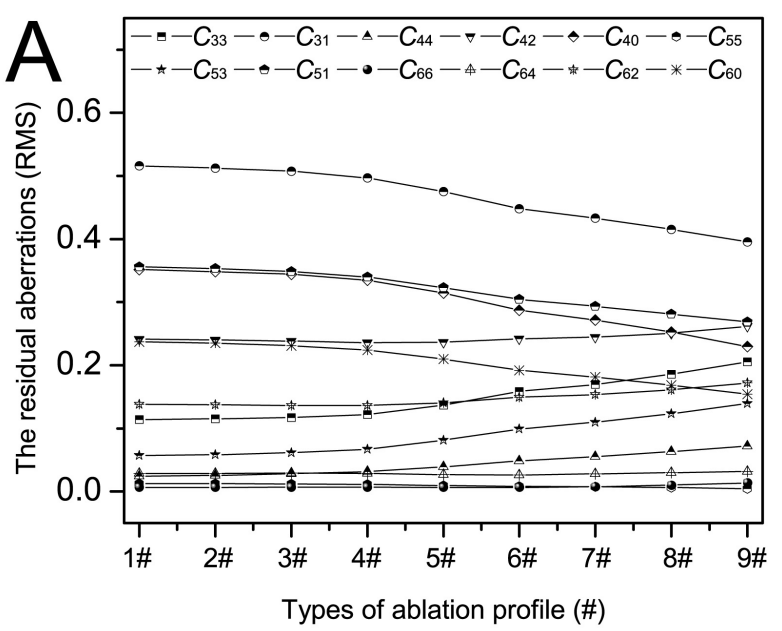

(a) For $-5.0 \mathrm{DS}-2.0 \mathrm{DC} \times 60^{\circ}$

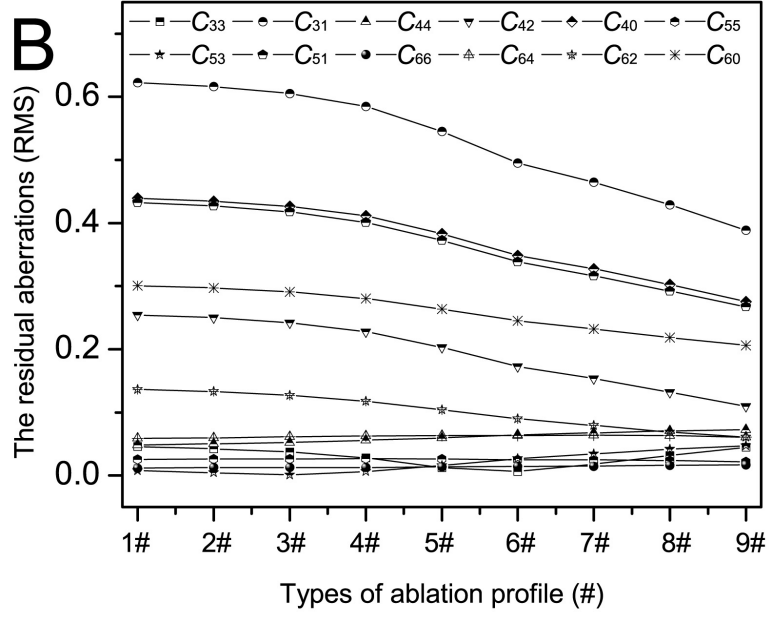

(b) For $-7.5 \mathrm{DS}-1.5 \mathrm{DC} \times 180^{\circ}$

FIG. 6 The residual higher-order aberrations for correcting myopia astigmatism versus the types of ablation profile in transition zone. The diameter of the optical zone is 6 mm and the width of transition zone $x_{0}$ is $1.05 \mathrm{~mm}$. The results reveal that the induced residual coma, spherical aberration and secondary coma decrease with the number of ablation profile for transition zone increasing.

The results indicate that the induced aberrations are distinctly different by comparison panel 5(a) with panel 5(b). The induced coma and sphere for profile\#1 shows relatively larger than those for profile \#9. In addition, the induced secondary astigmatism for profile \#9 increases as the increase of the translation of the centre of pupil. But for profile \#1, it increases first, and then decrease with increasing translation. In panel 5(a), the increasing induced trefoil as the increase of translation, and yet the types of profile show little impact of translation on the induced trefoil and its RMS value is smaller in panel 5(b). For secondary coma, the types of profile also have an important influence on the RMS values.

Furthermore, the Figure 6 shows the relationship between the residual aberrations and the types of ablation profile of aspheric transition zone for correcting myopia astigmatism when the translation remains constant being $0.4 \mathrm{~mm}$.

Figure 6 shows that the induced residual coma, spherical aberration and secondary coma decrease with the number of ablation profile for transition zone increasing, this is different from that for correcting pure hyperopia. Similarly, some aberration terms do not change with the increase of the number of ablation profile.

\section{DISCUSSION}

Clinically, the entrance pupil center is usually used as the ablation center. However, the human eye is a dynamic optical system and the entrance pupil center changes with accommodation and the level of illumination [13]. Therefore, subclinical ablation decentration usually occurs in refractive surgery. Lee found that the mean decentration was $0.23 \mathrm{~mm}$ in active eyetrackerassisted myopic photorefractive keratectomy (PRK) using the VISX STAR S4 laser with ActiveTrak (Abbott Medical Optics [AMO]) [14]. Therefore, the treatment decentration was added into our analysis. However, other studies have showed that static and dynamic decentrations may occur at same time during LASIK [15]. In our study, a limitation is that the dy- namic center errors during refractive surgery are not taken into account.

By applying transition zone, the corneal curvature can be continuous at the boundary between optical zone and transition zone, as well as at the boundary between transition zone and the unaltered zone. In addition, the haze may be confined to the wound edge, and the central cornea may remain relatively clear and provide good vision. Endl et al. revealed that corneal optical aberrations after photorefractive keratectomy with a larger ablation zone and a transition zone were less pronounced than those associated without transition zone [16]. In fact, the exact size, shape and the profile of transition zone have profound impacts on the residual optical aberrations and postoperative visual functions. In our previous researches, an ablation profile for transition zone was constructed, and results showed that the transition zone played a significant role on the induced aberrations in refractive surgery [17]. Our results were in agreement with the conclusion drawn from Kosaki et al. that the optimized aspheric transition zone (OATz) prole may decrease the surgically induced increase of higher order aberrations, especially spherical aberration, following LASIK [5]. Hori-Komai et al. indicated that patients treated with the OATz profiles had better visual quality as measured by contrast sensitivity and also had larger effective optical zones as compared with those treated by the conventional ablation profile [18]. Kermani et al. found that customized aspheric transition zones may help reduce induction of spherical aberration in refractive surgical correction of myopia and myopic astigmatism [19]. Seo et al. showed that higherorder aberrations after LASEK using a large optical zone with blend zone ablation were smaller than those associated with conventional ablation zone treatment in the scotopic condition. The larger zone with blend zone treatment may be a good surgical alternative for better visual outcomes in scotopic conditions [20]. In addition, the profile of transition zone can be designed for parabola and other profile, and the corresponding calculation results may be different from these aspheric transition zone profile. In fact, our results were also in agreement with the conclusion drawn from Arbelaez et al. that the main higher order aberration effects postoperatively 
(coma and spherical aberration) originated from decentration and "edge" effects, the strong local curvature change from optical zone to transition zone and from transition zone to the untreated cornea [6].

It is well-known that pupil size had a significant influence on the residual wavefront aberration RMS, especially for the condition of optical zone diameter larger than pupil size [21]. Also, our previous results indicated that the change in HOA root mean square and spherical aberration was significantly correlated with the optical zone to pupil ratio [7]. Therefore, matching problem between optical zone diameter and pupil size was very important in the design of refractive surgery. For a pure myopic correction, because the ablation depth of every point at the edge of optical zone is zero, the diameter of ablation zone is also $6 \mathrm{~mm}$ when the optical zone size is assumed to be $6 \mathrm{~mm}$ in this study. However, in practical clinic application, a larger ablation zone is designed in order to improve the postoperative optical performance and visual function, and the transition zone is required even though for a pure myopic correction [22]. In fact, an optimized transition zone can be result in an increase in the effective optical zone and improving good postoperative outcomes.

It is necessary to point out that the residual higher order aberrations may be decreased by optimizing ablation profiles of transition zone, but they cannot be eliminated. Because the removed power in transition zone is less than that in optical zone whatever ablation profiles of transition zone, and this results in the remaining some refractive errors in transition zone [23]. For a correction of myopia astigmatism, the profile \#1 shows the slightly larger induced coma and spherical aberration, which can be attributed to the stronger local curvature change from optical zone to transition zone and from transition zone to the untreated cornea [6]. On the contrary, for a correction of pure hyperopic correction, the profile \#1 indicates the slightly lower induced coma and spherical aberration. Therefore, the different ablation profiles of transition zone must be designed for hyperopic and myopic correction. On the other hand, the increase of the postoperative corneal asphericity and induction of spherical aberration could be explained partly by the ablation efficiency reduction due to laser oblique incidence [24]-[28]. Also, our previous results indicated that the effect of laser oblique incidence exerted an important influence on the residual wavefront aberrations [12]. So, the effect of oblique incidence was taken into account in this paper. In addition, ablation area irregularity may influence optical and functional outcomes of the refractive surgery and could be improved in regularity of the ablated surface by final smoothing $[29,30]$.

\section{CONCLUSION}

Based on the ablation profile for conventional ablation, the influence of aspheric transition zone on residual wavefront aberration was studied. Theoretical results revealed that the ablation profiles of transition zone exerted a significant influence on the residual wavefront aberrations. For a hyperopia correction, the induced coma is proportional to the translation of the centre of pupil and the profile \#9 shows a larger induced coma when the translation remains constant. In addition, the sign of the postoperative spherical aberration is negative and the profile \#9 shows that the induced spherical aberration has a larger RMS value than in other profiles. However, for a pure myopic correction, the ablation profiles of transition zone have no influence on the residual higher order aberrations. On the other hand, for a myopia astigmatism correction, the induced coma and spherical aberration for profile \#1 shows relatively larger RMS values than those for profile \#9. Therefore, an optimized transition zone can be result in an increase in the effective optical zone and improving good postoperative outcomes.

\section{ACKNOWLEDGEMENTS}

This research is supported by the National Natural Science Foundation of China (No. 61465010) and the Jiangxi nature science foundation (No. 20132BAB202009).

\section{References}

[1] S. MacRae, "Excimer ablation design and elliptical transition zones," J. Cataract Refract. Surg. 25, 1191-1197 (1999).

[2] M. S. Macsai, K. Stubbe, A. P. Beck, and Z. B. Ravage, "Effect of expanding the treatment zone of the Nidek EC-5000 laser on laser in situ keratomileusis outcomes," J. Cataract Refract. Surg 30, 2336-2343 (2004).

[3] K. Zhao, Y. Wang, T. Zuo, and H. Wang, "Multizone and transition zone photorefractive keratectomy for high myopia," J. Refract. Surg. 14, S222-225 (1998).

[4] T. Gamaly, "LASIK with the optimized aspheric transition zone and cross-cylinder technique for the treatment of astigmatism from 1.00 to 4.25 diopters," J. Refract. Surg. 25, S927-930 (2009).

[5] R. Kosaki, N. Maeda, H. Hayashi, T. Fujikado, and S. Okamoto, "Effect of NIDEK optimized aspheric transition zone ablation profile on higher order aberrations during LASIK for myopia," J. Refract. Surg. 25, 331-338 (2009).

[6] M. C. Arbelaez , C. Vidal, B. A. Jabri, and S. Arba Mosquera, "LASIK for myopia with Aspheric 'aberration neutral' ablations using the ESIRIS laser system," J. Refract. Surg. 25, 991-999 (2009).

[7] L. Fang, Y. Wang, and X. He, "Effect of pupil size on residual wavefront aberration with transition zone after customized laser refractive surgery," Opt. Express 21, 1404-1416 (2013).

[8] P. Padmanabhan, M. Mrochen, D. Viswanathan, and S. Basuthkar, "Wavefront aberrations in eyes with decentered ablations," J. Cataract Refract. Surg. 35, 695-702 (2009).

[9] N. Sakata, T. Tokunaga, K. Miyata, and T. Oshika, “Changes in contrast sensitivity function and ocular higher order aberration by conventional myopic photorefractive keratectomy," Jpn. J. Ophthalmol. 51, 347-352 (2007).

[10] N. Yamane, K. Miyata T. Samejima, T. Hiraoka, T. Kiuchi, F. Okamoto, Y. Hirohara, et al., "Ocular higher-order aberrations and contrast sensitivity after conventional laser in situ keratomileusis," J. Invest. Ophthalmol. Vis. Sci. 45 3986-3990 (2004).

[11] L. Wang, and D. D. Koch, "Residual higher-order aberrations caused by clinically measured cyclotorsional misalignment or decentration during wavefront-guided excimer laser corneal ablation," J. Cataract. Refract. Surg. 34, 2057-2062 (2008). 
[12] L. Fang, X. He, and F. Chen, "Theoretical analysis of wavefront aberration from treatment decentration with oblique incidence after conventional laser refractive surgery," Opt. Express 18, 22418-22431 (2010).

[13] L. Wu, X. Zhou, R. Chu, and Q. Wang, "Photoablation centration on the corneal optical center in myopic LASIK using AOV excimer laser," Eur. J. Ophthalmol. 19, 923-929 (2009).

[14] S. B. Lee, B. S. Hwang, and J. Lee, "Effects of decentration of photorefractive keratectomy on the induction of higher order wavefront aberrations," J. Refract. Surg. 26, 731-743 (2010).

[15] J. L. Febbraro, D. D. Koch, H. N. Khan, A. Saad, and D. Gatinel, "Detection of static cyclotorsion and compensation for dynamic cyclotorsion in laser in situ keratomileusis," J. Cataract. Refract. Surg. 36, 1718-1723 (2010).

[16] M. J. Endl, C. E. Martinez, S. D. Klyce, M. B. McDonald, S. J. Coorpender, R. A. Applegate, and H. C. Howland, "Effect of larger ablation zone and transition zone on corneal optical aberrations after photorefractive keratectomy," Arch. Ophthalmol 119, 1159-1164 (2001).

[17] L. Fang, Y. Wang, and X. He, "Theoretical analysis of wavefront aberration caused by treatment decentration and transition zone after custom myopic laser refractive surgery," J. Cataract Refract. Surg. (2013) article in press.

[18] Y. Hori-Komai, I. Toda, N. Asano-Kato, M. Ito, T. Yamamoto, and K. Tsubota, "Comparison of LASIK using the NIDEK EC-5000 optimized aspheric transition zone (OATz) and conventional ablation profile," J. Refract. Surg. 22, 546-555 (2006).

[19] 0. Kermani, K.Schmiedt, U. Oberheide, and G. Gerten, "Early results of nidek customized aspheric transition zones (CATz) in laser in situ keratomileusis," J. Refract. Surg. 19, S190-194 (2003).

[20] K. Y. Seo, J. B. Lee, J. J. Kang, E. S. Lee, and E. K. Kim, “Comparison of higher-order aberrations after LASEK with a $6.0 \mathrm{~mm}$ ablation zone and a $6.5 \mathrm{~mm}$ ablation zone with blend zone," J. Cataract. Refract. Surg. 30, 653-657 (2004).

[21] J. Bühren, C. Kühne, and T. Kohnen, "Influence of pupil and optical zone diameter on higher-order aberrations after wavefront-guided myopic LASIK," J. Cataract. Refract. Surg. 31, 2272-2280 (2005).
[22] M. Camellin, and S. Arba Mosquera, "Aspheric optical zones: the effective optical zone with the SCHWIND AMARIS," J. Refract. Surg. 27, 135-146 (2011).

[23] M. C. Corbett, S. Verma, D. P. O'Brart, K. M. Oliver, G. Heacock, and J. Marshall, "Effect of ablation profile on wound healing and visual performance 1 year after excimer laser photorefractive keratectomy," Br. J. Ophthalmol. 80, 224-234 (1996).

[24] J. R. Jiménez, F. Rodríguez-Marín, R. G. Anera, and L. Jiménez Del Barco, "Deviations of Lambert-Beer's law affect corneal refractive parameters after refractive surgery," Opt. Express 14, 5411-5417 (2006).

[25] Y. Kwon, and S. Bott, "Postsurgery corneal asphericity and spherical aberration due to ablation efficiency reduction and corneal remodelling in refractive surgeries," Eye 23, 1845-1850 (2009).

[26] Y. Kwon, M. Choi, and S. Bott, "Impact of ablation efficiency reduction on post-surgery corneal asphericity: simulation of the laser refractive surgery with a flying spot laser beam," Opt. Express 16, 11808-11821 (2008).

[27] S. Arba-Mosquera, and D. de Ortueta, "Ceometrical analysis of the loss of ablation efficiency at non-normal incidence," Opt. Express $16,3877-3895$ (2008).

[28] C. Dorronsoro, L. Remon, J. Merayo-Lloves, and S. Marcos, "Experimental evaluation of optimized ablation patterns for laser refractive surgery," Opt. Express 17, 15292-15307 (2009).

[29] P. Vinciguerra, M. Azzolini, P. Airaghi, P. Radice, and V. De Molfetta, "Effect of decreasing surface and interface irregularities after photorefractive keratectomy and laser in situ keratomileusis on optical and functional outcomes," J. Refract. Surg. 14, S199-203 (1998).

[30] P. Vinciguerra, F. I. Camesasca, and I. M. Torres, “Transition zone design and smoothing in custom laser-assisted subepithelial keratectomy," J. Cataract. Refract. Surg. 31, 39-47 (2005). 